\title{
Introduction to IT Enabled Collaboration in Developing Countries Minitrack
}

\author{
Xusen Cheng \\ University of International \\ Business and Economics, \\ Beijing, China \\ xusen.cheng@uibe.edu.cn
}

\author{
Xiangbin Yan \\ Harbin Institute of Technology, \\ Harbin, China \\ xbyan@hit.edu.cn
}

\author{
Deepinder Bajwa \\ Western Washington University \\ Bellingham, USA \\ deepinder.bajwa@wwu.edu
}

Collaboration theories and technologies have been applied and investigated in many countries and regions for various collaboration scenarios. As the world economy develops rapidly, collaboration in developing regions like East Europe, Asia, Africa, Central America, and South America have raised a lot of attention and the use of collaboration technologies continues to increase in countries in these regions. Despite these trends, for the most part, research on IT enabled collaboration in developing countries remains scarce. Our minitrack was introduced to fill this void in the literature. This is the third year that we are running this minitrack and as the submissions continue to increase each year, we hope that research on IT enabled collaboration in developing countries will gain popularity. After peer reviews, we accepted six papers for inclusion in the HICSS conference proceedings.

The first paper is "The Role of Information Exchange in Supply Chain Collaboration: A Case Study of a Vietnam Ship Parts Supplier" by Judith Molka-Danielsen, Bich Thi Ngoc Le, and Per Engelseth. In their paper, the authors examine collaborative supply chain strategies of a Vietnam Ship Parts Supplier.

The second paper is "An Object-oriented Workflow Modeling Schema Using Dataflow Analysis for Collaborative E-governance Platform" by Jingrui Ju, Luning Liu, and Yuqiang Feng. Based on collaborative e-governance platform, the authors propose a schema for e-governance platforms modeling according to an extended method of object-oriented workflow modeling using dataflow analysis.

The third paper is 'Employees' Collaborative Use of Green Information Systems" by Zhaojun Yang, Jun Sun, Yali Zhang, Ying Wang, and Lisha Cao. In this paper, the authors investigate the motivation, effort, and performance of collaborative GIS use in China.

The fourth paper is "Cross-Sector Collaboration and Information Integration in Local Government One-Stop Services Centers: The Experiences and Lessons from a Case Study in China" by Nan Zhang,
Zhikui Lu, and Yunyun Shou. In this paper, the authors address concerns with cross-sector collaboration and information integration through case study analysis.

The fifth paper is "Network Structure and User Roles of a Crowdsourcing Community - The Context of Social Innovations for a Development Project" by Simon Fuger, Robert Schimpf, Johann Füller, and Katja Hutter. In this paper, the authors apply Social network analysis (SNA) and network measures to visualize and interpret the network structure of the crowdsourcing community.

The sixth paper is "Individual Trust Development in Business Virtual Teams: An Experimental Study" by Xusen Cheng, Tingting Hou, Shixuan $\mathrm{Fu}$ and Jianshan Sun. In their research, the authors conducted a longitudinal case study on trust development in business virtual teams.

Collectively, these six papers focus on collaborative relationships in information technology enabled platforms investigated from the perspective of team virtual collaboration, collaboration in e-government systems, and collaboration in development projects. Based on survey, case studies, and design science, these papers contribute to the area of IT enabled collaboration for development and their practical implications.

Compared with the former two years, from the inception, the papers in this minitrack involve many facets of IT enabled collaborations in developing countries. We thank the authors for their submissions and acknowledge the reviewers for their feedback.

Moving forward, we would like to encourage researchers to explore other facets of IT enabled collaboration in developing countries including: behavior and design research on group decision making, negotiation, facilitation, and communication technologies for development. We would also like to welcome research on information systems, technologies, theories, process, methods, or models that could be transferred and applied from developed regions to developing regions. 